\title{
Iterative Optimization of Quantum Error Correcting Codes
}

\author{
M. Reimpell, R. F. Werner \\ Institut für Mathematische Physik, TU-Braunschweig, \\ Mendelssohnstraße 3, D-38106 Braunschweig, Germany
}

\begin{abstract}
We introduce a convergent iterative algorithm for finding the optimal coding and decoding operations for an arbitrary noisy quantum channel. This algorithm does not require any error syndrome to be corrected completely, and hence also finds codes outside the usual Knill-Laflamme definition of error correcting codes. The iteration is shown to improve the figure of merit "channel fidelity" in every step.
\end{abstract}

\section{INTRODUCTION}

From the beginning of the development of quantum information theory, it was recognized that without suitable error correcting procedures the fantastic promises of this new discipline, such as the exponential speedup in Shor's algorithm, or the possibility of long range quantum communication and secure key exchange would never be realizable. Therefore, the development of the first error correcting codes [1, 2] and the subsequent more systematic theory by Knill and Laflamme [3] were crucial achievements. It became clear that although naive classical ideas, like redundant transmission and majority rule decisions on the outputs, are ruled out by the no-cloning theorem, techniques from classical coding theory (e.g., additive codes) could be used to construct good quantum codes as well. The quantum codes constructed in this way share with their classical counterparts the combinatorial/algebraic flavor. They are designed to correct a certain finite dimensional subspace of errors, such as errors occurring on only a small number of the parallel channels employed. If the space of corrected errors is suitably chosen, such codes can also be used to correct generic small errors, i.e., one can show that any channel close to the ideal channel can be corrected with small overhead [4].

However, for errors of fixed finite size it is not at all clear that the special form of Knill-Laflamme codes allows the most efficient error correction. Alternative codes might not correct any error completely, but in exchange might improve correction of the errors ignored by the Knill-Laflamme codes, resulting in an improved overall performance. Consider, for example, the famous five bit code 5, 6], applied to the five-fold tensor product of a depolarizing qubit channel with a certain depolarization probability $p$. Figure 1 1 shows the fidelity achieved by this code as a function of $p$, together with the same parameter without any correction. For $p>1-\sqrt{2 / 3} \approx 18 \%$ the performance of the five bit code is actually worse than doing no correction operation at all. It seems implausible that the best code should jump from using all qubits to using only one at the crossover point, which suggests looking for better codes in that area.

In this Letter we develop a method which allows us to

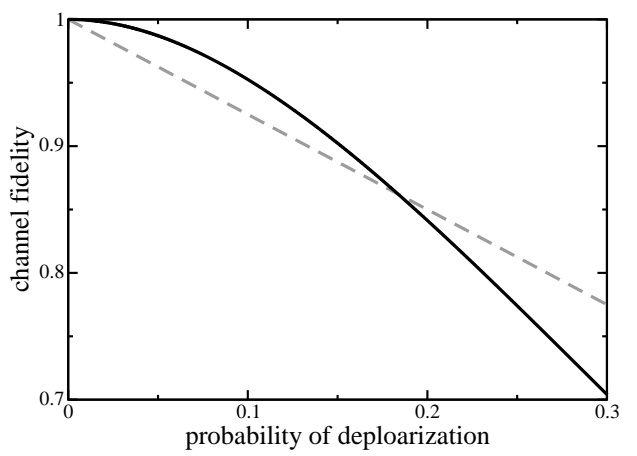

FIG. 1: Fidelity of the five bit code applied to 5-fold tensor product of the depolarizing qubit channel (solid line) compared to the fidelity of the depolarizing channel (dashed line).

search numerically for an optimal code adapted to arbitrary noise. Thus, for any given noisy channel $T$ (not necessarily a product $T=S^{\otimes n}$ of channels operating independently on $n$ smaller systems), we look for an encoding channel $E$ and a decoding channel $D$, with suitable domain and range, such that DTE comes as close as possible to the ideal channel on a fixed $d$-level system. In contrast to Knill-Laflamme theory we make no assumptions on the coding and decoding channels $E$ and $D$. The basis of the method is an iteration by which either $E$ or $D$ is changed, such that fidelity is improved in each step. The results are related to Knill-Laflamme theory as follows:

1. Surprisingly, the codes in Fig 1 turn out to be optimal already: up to the critical value of the depolarization probability the five-bit code is optimal, and beyond that the best way of using up to five bit encodings is to do nothing. However, this has little bearing on general channels, since the depolarizing channels are highly symmetric.

2. The encoding operation comes out to be an isometry even on random channels. This is a basic feature of Knill-Laflamme theory.

3. Sometimes the Knill-Laflamme theory applies, but not, as is usually done, to the correction of localized errors. Instead, certain non-localized errors 
are corrected. Such instances are also known in the decoherence-free subspace approach [7], and are reliably found by our method, since we do not use a tensor product decomposition of $T$ in the first place.

4. Sometimes Knill-Laflamme theory fails entirely in the sense that no error at all is corrected completely: although $E, D$ optimally correct a channel $T$, there might be no channel $T^{\prime}$ such that $D T^{\prime} E$ is a multiple of the identity.

\section{OVERVIEW OF THE METHOD}

Quantum information theory describes computation in terms of preparation, processing and measurements. We consider only finite dimensional quantum systems, i.e., systems whose observable algebra is of the form $\mathcal{B}(\mathcal{H})$, the linear operators on a finite dimensional Hilbert space $\mathcal{H}$. The quantum states, which physically describe the preparation process are given by density operators $\rho$ in $\mathcal{B}(\mathcal{H})$. Measurements are given by selfadjoint operators on $\mathcal{H}$, or, more generally by positive operator-valued measures. Processing operations, e.g. the free time evolution, a computation or a noisy transmission, are described by channels. These can either be considered as a modification of all subsequent measurements (Heisenberg picture), or as a modification of the preparation (Schrödinger picture). In this article we choose the latter option, i.e., channels are mathematically given by completely positive trace preserving operators $S: \mathcal{B}\left(\mathcal{H}_{1}\right) \rightarrow \mathcal{B}\left(\mathcal{H}_{2}\right)$, where $\mathcal{H}_{1}$ is the Hilbert space of the input systems, and $\mathcal{H}_{2}$ describes the output systems, and $S(\rho)$ is the state obtained by sending the input state $\rho$ through the channel. The encoding and decoding operations of an error correction scheme are also channels in this sense, with appropriate choices of input and output Hilbert spaces. Every channel $S$ has a Kraus representation $S(\rho)=\sum_{i} s_{i} \rho s_{i}^{*}$, with $s_{i}: \mathcal{H}_{1} \rightarrow \mathcal{H}_{2}$, and $\sum_{i} s_{i}^{*} s_{i}=\mathbb{1}$. When $\mathcal{H}_{1}=\mathcal{H}_{2}$, the 'noisiness' of $S$ is, loosely speaking, its distance from the ideal channel. There are many different ways of expressing this quantitatively. In this Letter we use a special case of Schumacher's Entanglement Fidelity 8], the channel fidelity. It is defined as

$$
\begin{aligned}
F_{C}(S) & =\langle\Omega|(\operatorname{id} \otimes S)(|\Omega\rangle\langle\Omega|)| \Omega\rangle \\
& =\left(\operatorname{dim} \mathcal{H}_{1}\right)^{-2} \sum_{i}\left|\operatorname{tr}\left(s_{i}\right)\right|^{2},
\end{aligned}
$$

where $|\Omega\rangle=\left(\operatorname{dim} \mathcal{H}_{1}\right)^{(-1 / 2)} \sum_{k}|k k\rangle$ is the standard maximally entangled unit vector in $\mathcal{H}_{1} \otimes \mathcal{H}_{1}$ and id is the identity channel on $\mathcal{B}\left(\mathcal{H}_{1}\right)$. This quantity is 1 if and only if the channel is ideal, and is directly related to the mean fidelity for pure input states [9].
The problem of finding an optimal code for a given channel $T: \mathcal{B}\left(\mathcal{H}_{1}\right) \rightarrow \mathcal{B}\left(\mathcal{H}_{2}\right)$ is now the construction of an encoding channel $E: \mathcal{B}\left(\mathcal{H}_{0}\right) \rightarrow \mathcal{B}\left(\mathcal{H}_{1}\right)$ and a decoding channel $D: \mathcal{B}\left(\mathcal{H}_{2}\right) \rightarrow \mathcal{B}\left(\mathcal{H}_{0}\right)$ such that $F_{C}(D T E)$ becomes maximal. This is always a fairly high dimensional search problem. For example, if $\mathcal{H}_{0}$ is a single qubit, and $T$ is the five-fold tensor power of a given noisy channel $\left(\operatorname{dim} \mathcal{H}_{1}=32\right)$, i.e., the case considered in figure 1 . the description of $D$ and $E$ together requires some 7000 parameters. General purpose optimization routines will usually choke on this, and there only is a chance if special properties of this variational problem can be brought to bear.

What we use in the present Letter is that the functionals $E \mapsto F_{C}(D T E)$ and $D \mapsto F_{C}(D T E)$ are both linear, and take positive values on completely positive operators. The iteration procedure described in the next section finds a maximum of any functional with these properties. The overall maximization then proceeds seesaw fashion, by applying the iteration first with a fixed random $E$, optimizing the fidelity over $D$, then fixing $D$ and optimizing $E$, and so on. Since every step of the iteration is proved to increase fidelity and each stable fixed point of the single iteration is a global maximum, this procedure is guaranteed to find at least a locally optimal pair of encoder and decoder.

\section{THE BASIC ITERATION}

The iteration we consider is a close relative of the power method for finding the eigenvector for the largest eigenvalue of a positive semi-definite matrix $A$. This method starts with a random unit vector $\phi_{0}$, and each step consists of applying $A$, and normalizing, i.e., $\phi_{n+1}=$ $A \phi_{n} /\left\|A \phi_{n}\right\|$. It is easy to see that the convergence of this algorithm is exponential with a rate determined by the gap to the next largest eigenvalue. Moreover, the inequality

$$
\frac{\left\langle\phi \mid A^{3} \phi\right\rangle}{\left\langle\phi \mid A^{2} \phi\right\rangle} \geq \frac{\langle\phi \mid A \phi\rangle}{\langle\phi \mid \phi\rangle},
$$

which is valid for any positive semi-definite Hilbert space operator $A$, shows that convergence is monotone, in the sense that $\left\langle\phi_{n} \mid A \phi_{n}\right\rangle$ is a non-decreasing sequence.

Suppose now that we want to find a channel $S$ maximizing a linear objective functional $f$, which is defined on arbitrary operators $S: \mathcal{B}\left(\mathcal{H}_{1}\right) \rightarrow \mathcal{B}\left(\mathcal{H}_{2}\right)$, and positive on all completely positive maps. Note that $f(S)$ is bilinear in the Kraus operators $s_{i}$ of $S$. So in a sense we will presently make precise, the objective functional $f$ is analogous to the matrix element $\left\langle\phi_{S} \mid \widetilde{F} \phi_{S}\right\rangle$ of a positive operator associated with $f$, where the vector $\phi_{S}$ corresponds to the set of Kraus operators $s_{i}$. In our iteration we apply the operator $\widetilde{F}$ to each Kraus operator and get a modified completely positive map. This will not 
be a channel, because it is not trace preserving. Hence we have to include a normalization step. Since the normalization of a completely positive map is given by an operator (not a scalar) one cannot simply "divide by the normalization". We show, however, how to do the normalization in such a way that the desirable features of the power method do carry over.

Let us now make these ideas precise. By $\mathcal{L}^{2}\left(\mathcal{H}_{1}, \mathcal{H}_{2}\right)$ we denote the space of Hilbert Schmidt operators from $\mathcal{H}_{1}$ to $\mathcal{H}_{2}$ with scalar product $\langle\langle x \mid y\rangle\rangle=\operatorname{tr}\left(x^{*} y\right)$. Then if $|\mu\rangle, \mu=1, \ldots, \operatorname{dim} \mathcal{H}_{1}$ denotes the vectors of a basis of $\mathcal{H}_{1}$, we associate with any map $S: \mathcal{B}\left(\mathcal{H}_{1}\right) \rightarrow \mathcal{B}\left(\mathcal{H}_{2}\right)$ an operator $\widetilde{S} \in \mathcal{B}\left(\mathcal{L}^{2}\left(\mathcal{H}_{1}, \mathcal{H}_{2}\right)\right)$ by

$$
\widetilde{S}(x)=\sum_{\mu \nu} S(|\mu\rangle\langle\nu|) x|\nu\rangle\langle\mu|,
$$

In fact, this is just a reshuffling of matrix elements since $\langle a|S(|\mu\rangle\langle\nu|)| b\rangle=\langle a|\widetilde{S}(|b\rangle\langle\nu|)| \mu\rangle$. The key feature of the correspondence $S \leftrightarrow \widetilde{S}$, also known as the Jamiolkowski duality, is that $S$ is completely positive if and only if $\widetilde{S}$ is a positive semi-definite operator on the Hilbert space $\mathcal{L}^{2}\left(\mathcal{H}_{1}, \mathcal{H}_{2}\right)$. Indeed, the Kraus decomposition of $S$ translates directly into

$$
\left.\widetilde{S}=\sum_{i}\left|s_{i}\right\rangle\right\rangle\left\langle\left\langle s_{i}\right|\right.
$$

and the operators with such a representation are precisely the positive semi-definite operators on $\mathcal{L}^{2}\left(\mathcal{H}_{1}, \mathcal{H}_{2}\right)$.

The objective functional $f(S)$ can now be written in terms of $\widetilde{S}$, and thus becomes a positive linear functional on the positive operators on $\mathcal{L}^{2}\left(\mathcal{H}_{1}, \mathcal{H}_{2}\right)$. But such functionals are themselves given by positive operators: there is a positive semi-definite $\widetilde{F}$ such that

$$
f(S)=\operatorname{tr}(\widetilde{F} \widetilde{S})=\sum_{i}\left\langle\left\langle s_{i} \mid \widetilde{F} s_{i}\right\rangle\right\rangle,
$$

where at the second equality we have inserted Eq. (4).

In each iteration we define a new completely positive map $S^{\prime}$ by applying $\widetilde{F}$ to each $s_{i}$, i.e., $s_{i}^{\prime}=\widetilde{F}\left(s_{i}\right)$, or

$$
\left.\widetilde{S}^{\prime}=\sum_{i} \widetilde{F}\left|s_{i}\right\rangle\right\rangle\left\langle\left\langle s_{i}\right| \widetilde{F}=\widetilde{F} \widetilde{S} \widetilde{F} .\right.
$$

Clearly, $S^{\prime}$ is usually not trace preserving. Instead, we have $\operatorname{tr}\left(S^{\prime}(\rho)\right)=\operatorname{tr}(M \rho)$, where

$$
M=\sum_{i}\left(s_{i}^{\prime}\right)^{*} s_{i}^{\prime}
$$

In order to normalize the channel we therefore multiply each $s_{i}^{\prime}$ with the suitable power of $M$ : if $M$ is nonsingular, we set $t_{i}=s_{i}^{\prime} M^{-1 / 2}$, so $\sum_{i} t_{i}^{*} t_{i}=\mathbb{1}$. These will be the Kraus operators of the next iterate $S_{+}$, i.e., the overall iteration step is

$$
S \mapsto S_{+}, \quad S_{+}(\rho)=S^{\prime}\left(M^{-1 / 2} \rho M^{-1 / 2}\right),
$$

with $S^{\prime}$ determined by Eq. (6). In the applications below $M$ is always invertible. But when $M$ is singular, we can still take $M^{-1 / 2}$ as the pseudo-inverse, and the channel $S_{+}$becomes normalized to a projection, i.e., it is trace preserving only for input density matrices on the support subspace of $M$ and annihilates density operators supported on the complement.

The properties of this iteration resemble those of the power method (which is, in fact, the special case $\left.\operatorname{dim} \mathcal{H}_{2}=1\right)$. Most importantly, one gets an improvement of the objective functional in every step: $f\left(S_{+}\right) \geq$ $f(S)$. The proof is based on inequality (2), for an operator $A$ depending on the normalization correction $M$, which hence changes in every step. As for the power method, there may be non-maximal fixed points of the iteration, corresponding to non-maximal eigenvalues of $A$. However, they are all unstable: a small random perturbation of such a fixed point is sufficient to get the iteration going again, finding strictly higher $f(S)$. Therefore, testing the stability of any fixed point found is included into the general algorithm.

We have proved that this "stabilized" iteration does converge to the global maximum, provided that the initial number of Kraus operators is sufficiently large to allow representation of arbitrary channels for the given dimensions. In that case convexity guarantees that there are no sub-optimal local maxima, and the linear stability analysis of the iteration shows that all stable points are indeed local maxima. Note that our iteration without stabilization never increases the number of Kraus operators, so we can also find local maxima with such a constraint, e.g., the constraint that encoding uses only one isometry.

\section{APPLICATION TO QUANTUM ERROR CORRECTION}

As mentioned above, we will optimize the overall fidelity of the corrected channel $F_{C}(D T E)$ by alternately fixing the decoding $D$ and optimizing the encoder $E$ by the iteration method, and fixing $E$ and optimizing $D$. Since both kinds of steps increase fidelity, this procedure converges to an optimum. All results reported below were computed by starting from various random initial configurations. The iteration was stopped when the gain of fidelity was below some threshold.

\section{Depolarizing Channel}

The procedure is applied to the depolarizing qubit channel with parameter $p$, i.e.,

$$
T_{p}(\rho)=p \operatorname{tr}(\rho) \frac{1}{2} \mathbb{1}+(1-p) \rho .
$$




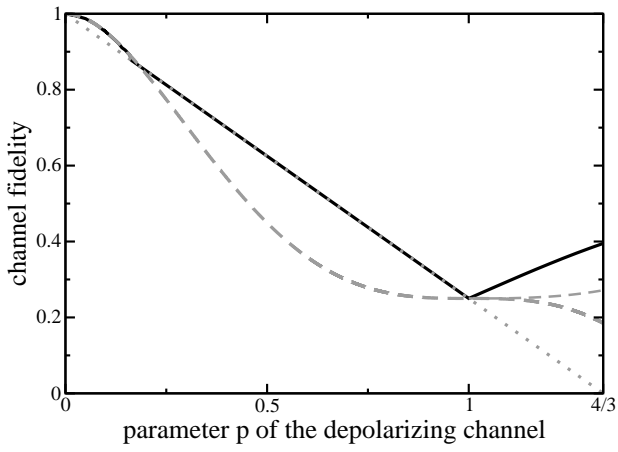

FIG. 2: Comparison of the channel fidelity of no error correction (dotted line), five bit code (dashed lines) and the iteration (solid line) applied to the 5-fold tensor product of depolarizing channel with parameter $p$. For $p>1$ also the fidelity for five-bit encoder combined with optimized decoder is shown.

For $0 \leq p \leq 1$ this channel totally depolarizes the input system with probability $p$ and leaves the input system untouched with probability $(1-p)$. The importance of this channel lies in its role as the worst case (the most mixed channel), whenever only a lower bound on the fidelity of a channel is known. The correction scheme for the depolarizing channel will then correct all such channels, to at least the same fidelity, even if further details are unknown. However, due to its high symmetry this channel is rather special (see Subsect. C below).

We will look at the fivefold tensor power of the depolarizing channel, since for fewer copies of the channel the optimal correction strategy turns out to do no correction at all, i. e., to copy the input to one of the output qubits and discard the rest. For five bits we have the standard five-bit stabilizer code [5, 6], which we denote by $\left(E_{5}, D_{5}\right)$. Its performance, given by the polynomial

$$
F_{C}\left(D_{5} T_{p}^{\otimes 5} E_{5}\right)=1-\frac{45}{8} p^{2}+\frac{75}{8} p^{3}-\frac{45}{8} p^{4}+\frac{9}{8} p^{5},
$$

is shown in Figure 2] Surprisingly, the optimal codes determined by our method fall exactly on the known lines: the five-bit code up to the cross-over point $p=$ $1-\sqrt{2 / 3} \approx 0.18$, and doing nothing for the range up to $p=1$. This is very surprising in view of the fact that the five bit code is not at all designed to give good results for large errors, but only to eliminate the linear term in (10).

\section{New Codes near the Universal Not}

Note that values $p>1$ in equation (9) are also admissible, as it defines a completely positive map for all $0 \leq p \leq 4 / 3$. For $p>1$ the channel correspond to a mixing of the totally depolarizing channel and the best possible approximation to the "universal not" channel
10]. In this range of $p$ our method does lead to a new type of code. By this we mean that in contrast to KnillLaflamme theory no error syndrome is corrected: there is no channel $T^{\prime}$ such that $D T^{\prime} E$ is a multiple of the identity, whereas any corrected error syndrome in the Knill-Laflamme theory would provide such $T^{\prime}$. We establish this result by our basic iteration, this time fixing $E, D$, and considering $T^{\prime}$ as the variable. On the other hand, by fixing $E=E_{5}$ and $T$, one can also check that it is not sufficient to just improve the decoder, and keep the five-bit-code encoding, as suggested by the analogous classical case of three bit flip channels with flip probability greater than $1 / 2$.

\section{Random Channels}

Moving away from the highly symmetric channels, we have considered random channels generated either with independent uniformly distributed entries, followed by normalization, or as convex combinations of such channels with the identity. In either case one sees that, generically, the optimized codes never correct a single syndrome. In contrast to the known limitations of KnillLaflamme codes, even for four encoding bits one often gets an improvement of the fidelity. More precisely, the fidelity after coding tends to increase (though often not by much) with every additional encoding qubit.

On the other hand, one feature of Knill-Laflamme theory is typically shared by the optimized codes: the encoding $E$ is isometric, i.e., it is given by a single Kraus operator. While it is known that this choice is asymptotically optimal (it suffices to get the same capacity as general encodings [4]), it is open whether it is also optimal for every fixed noisy channel, as suggested by our random search.

\section{ACKNOWLEDGEMENT}

We thank Koenraad M. R. Audenaert and Markus Grassl for discussions. Funding from Deutsche Forschungsgemeinschaft is gratefully acknowledged.

[1] P. W. Shor, Phys. Rev. A 52, R2493 (1995)

[2] A. M. Steane, Phys. Rev. Lett. 77, 793 (1996)

[3] E. Knill and R. Laflamme, Phys. Rev. A 55, 900 (1997)

[4] H. Barnum, E. Knill, M. A. Nielsen, IEEE Trans. Info. Theor. 46, 1317 (2000)

[5] R. Laflamme, C. Miquel, J. P. Paz and W. H. Zurek, Phys. Rev. Lett. 77, 198 (1996)

[6] C. H. Bennett, D. P. DiVincenzo, J. A. Smolin and W. K. Wootters, Phys. Rev. A 54, 3824 (1996)

[7] P. Zanardi, Phys. Rev. A 63, 012301 (2001) 
[8] B. Schumacher, Phys. Rev. A 54, 2614 (1996)

[9] M. Horodecki, P. Horodecki and R. Horodecki, Phys. Rev. A 60, 1888 (1999)
[10] V. Buzek, M. Hillery, R.F. Werner, Phys. Rev. A 60, R2626 (1999) 